NTCP for breast fibrosis

\title{
Normal tissue complication probability (NTCP) parameters for breast fibrosis: Pooled results from two randomised trials
}

\author{
Mukesh B. Mukesh ${ }^{\mathrm{a}, *}$, Emma Harris ${ }^{\mathrm{b}}$, Sandra Collette ${ }^{\mathrm{c}}$, Charlotte E. Coles ${ }^{\mathrm{a}}$, Harry Bartelink ${ }^{\mathrm{d}}$, \\ Jenny Wilkinson ${ }^{a}$, Philip M. Evans ${ }^{\text {, }}$, Peter Graham ${ }^{f}$, Jo Haviland ${ }^{g}$, Philip Poortmans ${ }^{\text {h }}$, \\ John Yarnold ${ }^{i}$, Raj Jena ${ }^{\text {a }}$
}

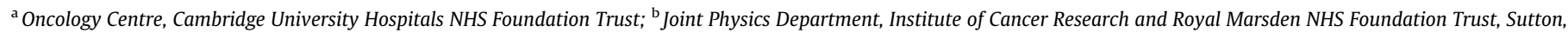

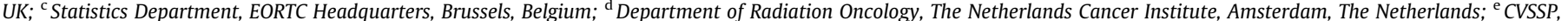

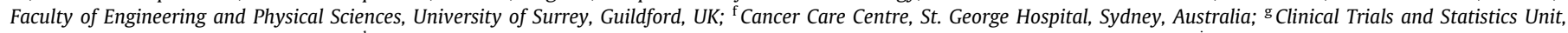
Institute of Cancer Research, Surrey, UK; ${ }^{\mathrm{h}}$ Department of Radiation Oncology, Dr. Bernard Verbeeten Instituut, Tilburg, The Netherlands; ${ }^{\mathrm{i}}$ Royal Marsden NHS Foundation Trust and Institute of Cancer Research, Sutton, UK

\section{A R T I C L E I N F O}

\section{Article history:}

Received 10 January 2013

Received in revised form 4 July 2013

Accepted 14 July 2013

Available online 13 August 2013

\section{Keywords:}

Breast radiotherapy

NTCP

Breast fibrosis

Breast conserving treatment

Late complications

\begin{abstract}
A B S T R A C T
Introduction: The dose-volume effect of radiation therapy on breast tissue is poorly understood. We estimate NTCP parameters for breast fibrosis after external beam radiotherapy.

Materials and methods: We pooled individual patient data of 5856 patients from 2 trials including whole breast irradiation followed with or without a boost. A two-compartment dose volume histogram model was used with boost volume as the first compartment and the remaining breast volume as second compartment. Results from START-pilot trial $(n=1410)$ were used to test the predicted models.

Results: $26.8 \%$ patients in the Cambridge trial (5 years) and $20.7 \%$ patients in the EORTC trial (10 years) developed moderate-severe breast fibrosis. The best fit NTCP parameters were $\mathrm{BEUD}_{3}(50)=136.4 \mathrm{~Gy}$, $\gamma 50=0.9$ and $n=0.011$ for the Niemierko model and $\operatorname{BEUD}_{3}(50)=132 \mathrm{~Gy}, m=0.35$ and $n=0.012$ for the Lyman Kutcher Burman model. The observed rates of fibrosis in the START-pilot trial agreed well with the predicted rates.

Conclusions: This large multi-centre pooled study suggests that the effect of volume parameter is small and the maximum RT dose is the most important parameter to influence breast fibrosis. A small value of volume parameter ' $n$ ' does not fit with the hypothesis that breast tissue is a parallel organ. However, this may reflect limitations in our current scoring system of fibrosis.
\end{abstract}

(c) 2013 Elsevier Ireland Ltd. All rights reserved. Radiotherapy and Oncology 108 (2013) 293-298
Radiation therapy (RT) has an established role in the management of early stage breast cancer to improve loco-regional control and overall survival [1]. However, a proportion of patients develop RT related complications including breast fibrosis, breast shrinkage and telangiectasia, which contribute to physical and psychological morbidity. Clinicians estimate the likelihood of a complication based on published literature and personal experience. The Emami et al. [2] seminal paper was among the first to provide a comprehensive review of radiation tolerance for normal tissues, estimating the tolerance doses $\left(\mathrm{TD}_{5}\right.$ and $\left.\mathrm{TD}_{50}\right)$ for whole, $2 / 3$ and $1 / 3$ organ irradiation. More recently, Quantitative Analyses of Normal Tissue Effects in the Clinic (QUANTEC) articles summarised the quantitative effects of RT dose and treatment volume on late normal tissue complications [3]. However, very few investigators have studied the radiation dose-volume effect for breast tissue [4-6].

\footnotetext{
* Corresponding author. Address: Oncology Centre, Box 193, Cambridge University Hospitals NHS Foundation Trust, Hills Road, Cambridge CB2 0QQ, UK.

E-mail addresses: mukesh.mukesh@addenbrookes.nhs.uk, drmukesh12@doctors.net.uk (M.B. Mukesh).
}

The influence of RT dose on late normal tissue complications is well established [7-9], however the effect of treated breast volume is unclear with conflicting reports in the literature [10]. The large EORTC 22881-10882 "boost versus no boost" trial reported higher breast fibrosis rates among patients treated with larger boost volumes on univariate analysis [11]. These results were hypothesis generating, consistent with a volume effect for breast fibrosis. Newer techniques aim to exploit a volume effect for breast tissue, including partial breast irradiation (PBI) [12], simultaneous integrated tumour bed boost (SIB) [13] and image guided RT (IGRT) [14], with the aim of reducing late normal tissue complications. As these techniques become part of routine practice, a better understanding of the dose-volume effect of radiation on breast tissue is required.

The normal tissue complication probability (NTCP) models can be used to estimate dose-volume effect by predicting the probability of a complication for a non-uniform irradiated organ. For the modelling exercise, one requires a dataset with diverse dose and volume data and a meaningful quantitative toxicity endpoint. 
The purpose of this study is to test the volume effect hypothesis and quantify the effect of volume parameter by estimating the NTCP model parameters for breast fibrosis as measured by induration score. Fibrosis is a common sequela of breast RT and adversely affects overall cosmesis, it can be assessed using a scoring system and is likely to impact on patient physical and psychological wellbeing [15]. Individual patient data from randomised controlled trials (RCTs) provide the most robust data on RT dose and toxicity. Additionally, pooling of data from different RCTs increases the diversity of the dataset and the generalisation of results to the wider population [16]. Hence, the individual patient data from two large RCTs were pooled together: EORTC 22881-10882 "boost versus no boost" trial $[8,9]$ and the Cambridge Breast IMRT trial $[17,18]$. To our knowledge, no other dataset of this magnitude has previously been pooled for the purpose of NTCP modelling for breast tissue.

\section{Materials and methods}

\section{Patient cohort and toxicity scoring}

Cambridge Breast IMRT trial [17,18]: This single centre trial recruited 1145 patients with invasive breast cancer (stage T1-T3N0$1 \mathrm{M} 0$ ) or ductal carcinoma in situ who received breast conserving therapy (BCT). All patients received $40 \mathrm{~Gy}$ in 15 fractions over 3 weeks to the whole breast followed by an electron tumour bed boost of $9 \mathrm{~Gy}$ in 3 fractions over 3 days in selected cases $(n=728)$. Breast fibrosis was clinically assessed at 2 and 5 years after completion of RT and scored on a four point scale $(0=$ none, $1=\mathrm{a}$ little, $2=$ quite $\mathrm{a}$ bit $(\sim$ moderate $)$ and $3=$ very much ( $\sim$ severe)).

EORTC 22881-10882 "boost versus no boost" trial [8,9]: This multi-centre trial recruited 5569 patients with invasive breast cancer (stage T1-T2N0-1M0) who received BCT. All patients received $50 \mathrm{~Gy}$ in 25 fractions over 5 weeks to the whole breast and were randomised between no boost $(n=2657), 10 \mathrm{~Gy}$ in 5 fractions boost ( $n=126), 16 \mathrm{~Gy}$ in 8 fractions boost $(n=2661)$ and $26 \mathrm{~Gy}$ in 13 fractions boost $(n=125)$. Electrons $(63 \%)$, photons $(29 \%)$ and low dose rate brachytherapy (9\%) were used to deliver the boost dose. Breast fibrosis was clinically assessed and scored on a four point scale ( 1 = none, $2=$ minor, $3=$ moderate, and $4=$ severe) at every follow up visit.

The brachytherapy technique can lead to significant dose heterogeneity and its boost volumes are usually much smaller than external beam techniques [19]. Hence, patients with brachytherapy boost were excluded from the analysis as were patients with missing data/toxicity score (Cambridge trial: 571 and EORTC trial: 275).

\section{Dose-volume data}

The accuracy with which NTCP model parameters can be estimated depends on the quality of both the dosimetric information and clinical follow up data. The late toxicity scores and boost volumes were recorded in the trials but limited dose-distribution data were available. Therefore, a more simplistic two-compartment dose-volume histogram (DVH) model was used. The first step of the DVH was the tumour bed volume receiving whole breast dose plus boost dose and the second step of the DVH was the remaining breast volume (whole breast volume minus tumour bed volume) receiving whole breast dose only.

Whole breast volume was only recorded in the Cambridge trial. Hence, a Monte Carlo (MC) simulation method was used to generate breast volume data for the EORTC patients. The MC simulation used the breast volume distribution from the Cambridge trial and an acceptance-rejection test of boost/breast volume ratio between
5-40\% (the range of boost volume to breast volume ratio observed in the Cambridge data). It was assumed that the distribution of breast volume and boost/breast volume ratio in the EORTC trial is the same as in the Cambridge trial.

\section{NTCP modelling}

Two radiobiological models were used: Lyman Kutcher Burman (LKB) model [20] and the Niemierko model [21]. Both models assume that for whole or partial organ irradiation, the dose-response curve follows a basic sigmoid shape. Full details of the mathematical modelling are given in appendix 1.

\section{Estimation of NTCP parameters with 95\% confidence interval}

A Maximum Likelihood Estimation (MLE) method [22] was used to find the best fit values of the model parameters (BEUD50 [biologically equivalent uniform dose leading to $50 \%$ toxicity], $\gamma 50 / \mathrm{m}$ [steepness of the dose-response curve] and $n$ [volume parameter]). A $n$ value closer to one suggests that the organ has a parallel architecture with a strong volume dependence whilst a value of $n$ closer to zero suggests that the organ has a serial architecture with no volume dependence on late complication rate. A full sequential parameter search was performed with the following parameter constrains: $\mathrm{BEUD}_{3}(0-150), n(0.01-1.0), \gamma 50$ (0.5-3.0) and $m$ (0.1-0.8). The $95 \%$ confidence intervals $(\mathrm{CI})$ for the optimally fit parameters were obtained using the Profile Likelihood Estimation method [23].

\section{Goodness of fit estimation}

Results from the START-pilot trial [24] were used to independently assess the goodness of fit of the predicted NTCP models. The START-pilot trial randomised 1410 patients into one of three whole breast RT dose fractionations: $50 \mathrm{~Gy}$ in 25 fractions or $39 \mathrm{~Gy}$ in 13 fractions or $42.9 \mathrm{~Gy}$ in 13 fractions. Patients were also sub-randomised for tumour bed boost to a dose of $14 \mathrm{~Gy}$ in $7 \mathrm{frac}-$ tions using electrons. Breast induration was clinically assessed at annual follow-up and scored on a four point scale $(0=$ none, $1=$ mild, $2=$ moderate and $3=$ marked $\{\sim$ severe $\})$. Summative data on moderate and severe breast induration at five years were used for all three whole breast dose fractionations with and without boost for the goodness of fit estimation. The goodness-of-fit statistic was obtained by calculating the Pearson chi-square statistic $\left(\chi^{2}\right)$ from the observed and predicted rates of breast fibrosis.

\section{Results}

Individual dose-volume and toxicity data of 574 patients (50\%) from the Cambridge trial and 5282 patients (95\%) from the EORTC trial were available for the NTCP modelling. 26.8\% (154/574) patients developed moderate-severe breast fibrosis by 5 years in the Cambridge trial and 20.7\% (1096/5282) patients developed moderate-severe breast fibrosis by 10 years in the EORTC trial. The patient's RT dose volume characteristics are summarised in Table 1.

Using the MLE method, the best fit NTCP parameters for the Niemierko model were $\mathrm{BEUD}_{3}(50)=136.4 \mathrm{~Gy}, \gamma 50=0.9$ and $n=0.011$. The $95 \% \mathrm{CI}$ for parameters were $\mathrm{BEUD}_{3}(50)=132.8-140 \mathrm{~Gy}$, $\gamma 50=0.84-0.97$ and $n=0.01-0.03$. For the LKB model, the best fit parameters were $\mathrm{BEUD}_{3}(50)=132 \mathrm{~Gy}, m=0.35$ and $n=0.012$ with $95 \% \mathrm{CI}$ of $\mathrm{BEUD}_{3}(50)=128.8-135.6 \mathrm{~Gy}, \quad m=0.326-0.374$ and $n=0.01-0.03$. Both models imply that the risk of moderate-severe breast fibrosis is strongly associated with RT dose and the effect of the volume parameter is small. Using an $\alpha / \beta$ of $3 \mathrm{~Gy}$ and $n=0.01$, EQD2 (the equivalent dose in $2 \mathrm{~Gy}$ per fraction) leading to $50 \%$ 
Table 1

Dose-volume characteristics from the Cambridge and the EORTC dataset used for the NTCP model.

\begin{tabular}{|c|c|c|c|}
\hline & Number of patients & Mean boost volume (range) & Moderate-severe fibrosis rates \\
\hline \multicolumn{4}{|c|}{ Cambridge dataset (assessed at 5-year) } \\
\hline No boost & 235 & - & $40 / 235(17 \%)$ \\
\hline Boost & 339 & $161.2(33.6-540 \mathrm{cc})$ & $114 / 339(33.6 \%)$ \\
\hline \multicolumn{4}{|c|}{ EORTC dataset (cumulative incidence at 10 years)* } \\
\hline No boost & 2656 & - & $341 / 2656(12.8 \%)$ \\
\hline$\geqslant 6 \mathrm{~Gy}$ to $<10 \mathrm{~Gy}$ & 6 & $238(108-372 \mathrm{cc})$ & $1 / 6(16.7 \%)$ \\
\hline $10 \mathrm{~Gy}$ & 117 & 204.7 (42-1176 cc) & $28 / 117(23.9 \%)$ \\
\hline $12 \mathrm{~Gy}$ & 31 & $185.9(48-606 \mathrm{cc})$ & $11 / 31(35.5 \%)$ \\
\hline $14 \mathrm{~Gy}$ & 93 & $273.4(48-735 \mathrm{cc})$ & $23 / 93(24.7 \%)$ \\
\hline $16 \mathrm{~Gy}$ & 2257 & $209(22-1386 \mathrm{cc})$ & $635 / 2257(28.1 \%)$ \\
\hline$>16 \mathrm{~Gy}$ to $\leqslant 20 \mathrm{~Gy}$ & 39 & $193.1(52-630 \mathrm{cc})$ & $9 / 39(23.1 \%)$ \\
\hline $26 \mathrm{~Gy}$ & 83 & 198.5 (43-630 cc) & $48 / 83(57.8 \%)$ \\
\hline
\end{tabular}

The NTCP parameter estimation was based on the actual tumour bed boost dose delivered and not on the intention to treat boost dose.

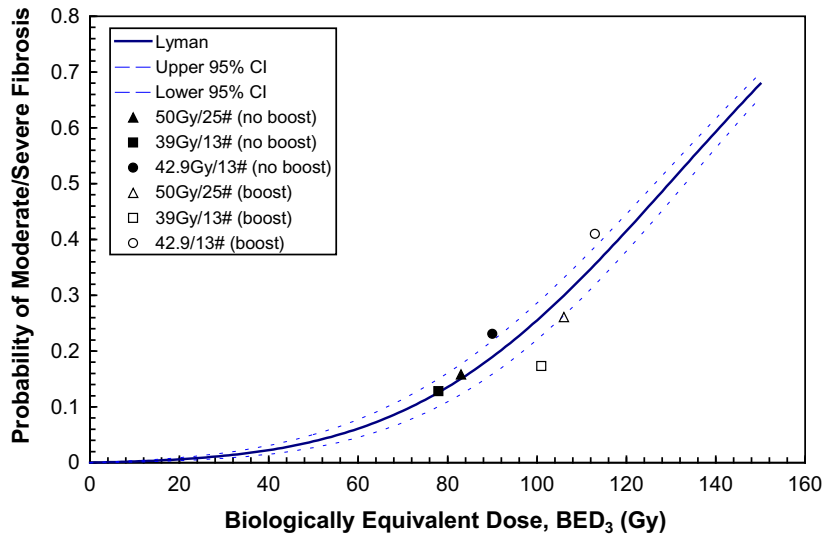

Fig. 1. Lyman Kutcher Burman model - the probability of moderate-severe breast fibrosis versus biological equivalent dose using $\alpha / \beta$ of $3 \mathrm{~Gy}\left(\mathrm{BED}_{3}\right)$. The solid line is based on the best fit parameters $\left(\mathrm{BED}_{3}=132 \mathrm{~Gy}\right.$ and $\left.m=0.35\right)$ and the dashed lines are upper and lower $95 \% \mathrm{CI}$. The summative toxicity data of the three dose fractionations \pm boost at five years from the START pilot trial are plotted.

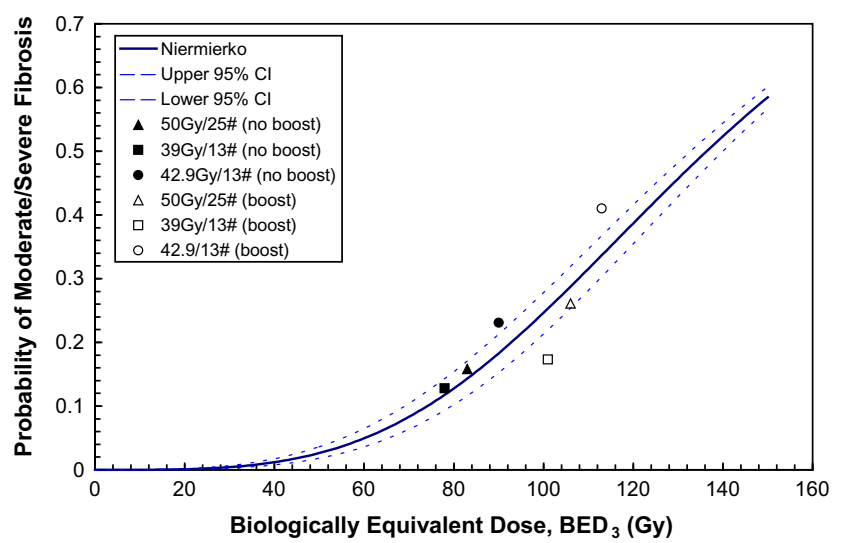

Fig. 2. Niemierko model - the probability of moderate-severe breast fibrosis versus biological equivalent dose using $\alpha / \beta$ of $3 \mathrm{~Gy}\left(\mathrm{BED}_{3}\right)$. The solid line is based on the best fit parameters $\left(\mathrm{BED}_{3}=136.4 \mathrm{~Gy}\right.$ and $\left.\gamma 50=0.9\right)$ and the dashed lines are upper and lower $95 \% \mathrm{CI}$. The summative toxicity data of the three dose fractionations \pm boost at five years from the START pilot trial are plotted.

probability of moderate-severe breast fibrosis were $79.2 \mathrm{~Gy}$ and $81.8 \mathrm{~Gy}$ for the LKB and Niemierko model, respectively.

The observed rates of moderate-severe induration in the START pilot trial were in good agreement with the predicted rates of fibrosis using the LKB model (Fig. 1) and the Niemierko model (Fig. 2). Using the Pearson chi-square test with 5 degrees of freedom, the $\chi^{2}$ was $0.053(p=0.95)$ for the LKB model and $\chi^{2}$ was $0.058(p=0.95)$ for the Niemierko model suggesting a good fit of the models.

\section{Discussion}

A better understanding of the dose-volume effect for breast tissue is timely as many patients now receive non-uniform breast irradiation in the form of accelerated PBI, SIB and risk adapted RT $[12,13,25,26]$. The EORTC 22881-10882 trial breast fibrosis nomogram showed a strong association between RT dose and fibrosis, with large boost volumes as a prognostic factor on univariate analysis only [11]. The purpose of this study was to specifically look at the volume effect by developing a predictive NTCP model. This was approached by pooling individual data from two large prospective trials (5856 patients), that offered robust information on RT dose, boost volume and late toxicity.

Using the MLE method, the volume parameter ' $n$ ' was close to zero for both the LKB model and the Niemierko model. This suggests that for moderate-severe fibrosis, the breast tissue behaves as a serial organ and the maximum RT dose is most predictive of the complication. The summative data of 1410 patients from an independent dataset with six RT dose levels had a good fit on both the LKB and Niemierko models (Figs. 1 and 2).

Parameter correlation leads to uncertainty of parameter estimates, independent of the size and diversity of the dataset [27]. An effective method to decrease the uncertainty is fixing one or more model parameters. Hence the $\alpha / \beta$ was fixed as $3 \mathrm{~Gy}$ in the study based on the previously published literature [24]. There is no evidence to suggest the superiority of one model over another [28]. However, similar values of the estimated parameters from the two models strengthen the results of this study.

Three other studies have previously estimated the NTCP parameters for breast fibrosis and these results are summarised in Table 2 . Borger et al. [4] model was based on 404 patients treated with WBI ( $50 \mathrm{~Gy}$ in 25 fractions over 5 weeks) followed by low dose rate Iridium-192 based tumour bed boost (15-25 Gy). BEUD was calculated using $\alpha / \beta$ of $2 \mathrm{~Gy}$ and repair half-time of $1.5 \mathrm{~h}$. The implant positions were re-constructed on the available radiographs and dosevolume calculations were performed. The best fit NTCP parameters in the study were TD50 $=72 \mathrm{~Gy}$ and $n=0.16 \pm 0.04$. Though informative, the model parameters were estimated from patients with brachytherapy boost alone. It is not evident to compare parameters generated from brachytherapy to external beam techniques due to the difference in dose distribution and a possible different radiobiological effect. For this reason, patients with brachytherapy boost were excluded in the current study. Avanzo et al. [5] estimated the best fit parameters for the model using average dosimetric parameters (prescription dose, fraction dose, median follow up 
Table 2

Summarised results of the best fit NTCP parameters for moderate-severe breast fibrosis.

\begin{tabular}{|c|c|c|c|c|c|}
\hline & Number of patients & $\mathrm{BEUD}_{3}(50)$ & $\gamma 50$ & $m$ & $n$ \\
\hline Borger et al. [4] & 404 & $\begin{array}{l}\mathrm{NTD}_{50}=72 \mathrm{~Gy} \\
(\alpha / \beta=2 \mathrm{~Gy}) \\
\left(t_{1 / 2}=1.5 \mathrm{~h}\right)\end{array}$ & & & 0.16 \\
\hline \multicolumn{6}{|l|}{ Alexander et al. ${ }^{*}[6]$} \\
\hline LKB model & 1546 & 104 Gy & - & 0.27 & 0.78 \\
\hline Relative seriality model & & 104 Gy & 1.47 & & $(s=0.12)$ \\
\hline \multicolumn{6}{|l|}{ Avanzo et al. ${ }^{*}[5]$} \\
\hline With repair correction $\left(\left(t_{1 / 2}=4.4 \mathrm{~h}\right)\right.$ & 2562 & $105.8 \mathrm{~Gy}$ & - & 0.22 & 0.15 \\
\hline Without repair correction & & $107.2 \mathrm{~Gy}$ & & 0.22 & 0.06 \\
\hline \multicolumn{6}{|l|}{ Current study } \\
\hline LKB model & 5856 & 132 Gy & & 0.35 & 0.012 \\
\hline Niemierko model & & 136.4 Gy & 0.9 & & 0.011 \\
\hline
\end{tabular}

NTD: Normalised total dose.

$\operatorname{BEUD}_{3}(50)$ : Biologically equivalent uniform dose using $\alpha / \beta$ of $3 \mathrm{~Gy}$.

$\gamma 50 / \mathrm{m}$ : Slope of the dose response curve.

$n$ : Volume parameter.

$t_{1 / 2}$ : Repair half-time.

$s$ : Describes the serial/parallel architecture of the organ. A value closer to 1 indicates a serial structure and a value closer to 0 indicates a parallel structure.

These studies used summative dosimetric and toxicity data.

and dose-volume data) from three WBI studies without boost and four external beam PBI studies. Three PBI studies used twice daily fractionation, and BEUD calculations included a repair half-time of $4.4 \mathrm{~h}$ in the model. As the median follow up of the PBI studies was short (1.3-4.2 years), a latency function correction was included. The parameters were estimated using weighted least square method, with the number of patients in each dataset as weights. The parameters for moderate-severe breast fibrosis model were BEUD50 $=105.8, n=0.15$ and $m=0.22$. The authors acknowledged that the gold standard approach to estimate NTCP parameters is the use of individual dosimetric data/clinical outcome. MLE method based parameter estimates are also more precise as compared to weighted least square method [29].

On the contrary, Alexander et al. [6] reported a strong effect of volume parameter on breast fibrosis. This study included summative data of 806 patients from the START- pilot trial [24], 590 patients from a Germany study [30] and 150 post-mastectomy patients treated during the 1960s [31]. All patients received WBI and no partial volume data were available for the fitting analysis. The dose-volume data were generated using an anthropomorphic phantom and parameters were estimated for a relative seriality model and Lyman model. The study suggested a parallel architecture for breast tissue with a strong volume effect on breast fibrosis $(n=0.78)$. However, these results cannot be generalised for several reasons:

(a) The study did not make allowance for the tumour bed boost doses (additional RT dose) in the models.

(b) The toxicity outcome used is different between the studies. The START-pilot and German study assessed breast fibrosis on clinical examination, whereas the post-mastectomy study scored fibrosis on photographs.

(c) The planning techniques for post-mastectomy study (1960s) would be considered as outdated by present standards. One would also expect different NTCP parameters for breast fibrosis after BCS and tissue fibrosis after mastectomy.

(d) The study corrected time latency in BCT study (START-pilot \& German) based on the results of the historic post-mastectomy series.

Overall, most studies have indicated a small volume effect for breast fibrosis. There are several possible reasons to explain the difficulty in demonstrating the effects of volume parameter for breast fibrosis. Breast fibrosis may represent a focal RT effect, with the maximum RT dose as the most predictive factor. It is also possible that our current scoring methods for breast fibrosis are not sensitive to the volume effect. Breast fibrosis is often graded as mild-severe based on the severity; however the scoring system does not take into account the extent of fibrosis i.e. small discrete region of fibrosis and widespread region of fibrosis are potentially scored alike. It has been suggested that NTCP parameters are influenced by the severity of measured toxicity [32]. For rectum, Rancati et al. estimated the best fit ' $n$ ' parameter was 0.23 for $\geqslant$ grade 2 rectal bleeding, which decreased to 0.06 when only severe rectal bleeding (grade 3 ) was considered [32]. It is plausible that a volume effect for breast tissue may have been seen for mild fibrosis, but this endpoint was considered to be of less clinical significance. Apart from RT parameters, breast fibrosis can also be influenced by surgical techniques [33] and systemic therapy [34], which are not accounted for in the mathematical model.

There is a need to investigate quantitative methods, which define both the severity and extent of breast fibrosis. The use of patient-reported toxicity scoring for NTCP modelling may also be useful. A small area of fibrosis in the breast may not be perceived as toxicity by the patient, whereas a large area of fibrosis in a small breast is likely to be considered as significant toxicity by the patient. Hence, patient-reported breast fibrosis scoring may be more sensitive to the change in treatment volume. Other toxicity endpoints like photographic assessed breast shrinkage may also be more sensitive to the volume effect as it represents global organ effect, is more objective and scored independent of surgical changes. The current study focused on breast fibrosis as photographic assessment and patient reported scoring were not available for the majority of the patients included in the study $[35,36]$.

\section{Limitations}

It is recognised that there are several limitations of this study. One of the intrinsic difficulties in modelling for breast tissue is the lack of detailed dosimetric data. A two-compartment DVH was used with the assumption that a homogeneous dose was delivered to the breast during WBI. The EORTC whole breast volume data were generated using MC simulation, using parameters from the Cambridge trial. It is clear that using simulated data for the EORTC patients can lead to large uncertainties. A plot of boost volume against moderate-severe fibrosis suggests that the volume effect is likely to be weak (Appendix Fig. 3) and the model parameters will not be affected by the distribution of the simulated 
breast volumes. To test this hypothesis, ten additional breast volume datasets were generated for the EORTC patients using the MC method. Furthermore, the variance of the first two simulated datasets was changed by 0.5 and 2 times the original value. Repeat simulations and changing the variance of breast volume distribution did not significantly change the estimated NTCP parameters (in keeping with weak volume effect). Simulation techniques are a powerful tool for modelling exercise. However, when applying simulated parameter from one population to the outcome data of another population, it is assumed that the two populations are identical. This may not be true in all cases.

Other limitations of the study include the use of both photons and electron boost modalities without any correction for their different radiobiological effectiveness (RBE). Bentzen et al. [37] previously reported RBE for electrons was 0.88 relative to photons at $4.1 \mathrm{~mm}$ depth. As the RBE difference at depths other than $4.1 \mathrm{~mm}$ is unknown, no attempts were made to correct for this. The duration of follow up was different between the EORTC (10 years) and Cambridge datasets (5 years). However, no suitable adjustment could be made in the MLE method for latency. In addition, current literature indicates that the majority of the breast fibrosis events take place by five years time point [11]. For this analysis, the score for fibrosis was used independent from the site in the breast (boost area or elsewhere). It is not expected to influence on our results, as it is most often located at the boost area (where the highest dose is given). Moreover, the worst score ever was reported. Although improvement of fibrosis is not expected, erroneous scoring of oedema early after treatment might be possible. Large breast volume has been reported as an independent risk factor for breast shrinkage and change in breast appearance $[38,39]$. However, one of the inherent limitations with NTCP modelling exercise is that it is driven by partial dose volume data, independent of whole organ volume. Apart from dose volume parameter, other patient (smoking, diabetes), treatment (type of surgery, chemotherapy, endocrine therapy and post-operative complications) and genetic factors also influence on breast fibrosis [11]. These factors were not available for the analysis in the current study.

\section{Conclusions}

This large multi-centre pooled study suggests that the effect of volume parameter is small and the maximum RT dose is the most important parameter to influence late breast fibrosis. However, this may reflect limitations in our current scoring system. Other RT associated complications should also be analysed to determine the effects of dose-volume parameters and patient-reported outcomes should complement clinician score-based models in the future. Inclusion of other clinical factors is desirable for future NTCP modelling work.

\section{Funding sources}

Dr. Mukesh B. Mukesh and Dr. Emma Harris are funded by the Efficacy and Mechanism Evaluation programme, Medical Research Council, UK (Grant no.: 09/150/16).

Dr. Charlotte Coles is supported by the Cambridge National Institute of Health Research Biomedical Research Centre.

Dr. Sandra Collette (EORTC Headquarters) was funded by the Fonds Cancer (FOCA) from Belgium.

\section{Disclaimer}

This report is independent research commissioned by the National Institute for Health Research, UK. The views expressed in this publication are those of the authors and not necessarily those of the NHS, the National Institute for Health Research or the Department of Health.

\section{Conflict of interest}

None.

\section{Acknowledgments}

We will like to thank Dr. Sarah Gulliford and Dr. Laurence Collette for their helpful advice and comments and Sue Tabor for helping with data collection.

\section{Appendix A. Supplementary data}

Supplementary data associated with this article can be found, in the online version, at http://dx.doi.org/10.1016/j.radonc.2013.07. 006.

\section{References}

[1] Darby S, McGale P, Correa C, et al. Effect of radiotherapy after breastconserving surgery on 10-year recurrence and 15-year breast cancer death: meta-analysis of individual patient data for 10,801 women in 17 randomised trials. Lancet 2011;378:1707-16.

[2] Emami B, Lyman J, Brown A, et al. Tolerance of normal tissue to therapeutic irradiation. Int J Radiat Oncol Biol Phys 1991;21:109-22.

[3] Marks LB, Yorke ED, Jackson A, et al. Use of normal tissue complication probability models in the clinic. Int J Radiat Oncol Biol Phys 2010;76:S9-S10.

[4] Borger JH, Kemperman H, Smitt HS, et al. Dose and volume effects on fibrosis after breast conservation therapy. Int J Radiat Oncol Biol Phys 1994;30: 1073-81.

[5] Avanzo M, Stancanello J, Trovo M, et al. Complication probability model for subcutaneous fibrosis based on published data of partial and whole breast irradiation. Phys Med 2012;28:296-306.

[6] Alexander MA, Brooks WA, Blake SW. Normal tissue complication probability modelling of tissue fibrosis following breast radiotherapy. Phys Med Biol 2007;52:1831-43.

[7] Van Limbergen E, Rijnders A, van der Schueren E, Lerut T, Christiaens R. Cosmetic evaluation of breast conserving treatment for mammary cancer. 2. A quantitative analysis of the influence of radiation dose, fractionation schedules and surgical treatment techniques on cosmetic results. Radiother Oncol 1989:16:253-67.

[8] Bartelink H, Horiot JC, Poortmans PM, et al. Impact of a higher radiation dose on local control and survival in breast-conserving therapy of early breast cancer: 10-year results of the randomized boost versus no boost EORTC 22881-10882 trial. J Clin Oncol 2007;25:3259-65.

[9] Poortmans PM, Collette L, Horiot JC, et al. Impact of the boost dose of $10 \mathrm{~Gy}$ versus $26 \mathrm{~Gy}$ in patients with early stage breast cancer after a microscopically incomplete lumpectomy: 10-year results of the randomised EORTC boost trial. Radiother Oncol 2009;90:80-5.

[10] Mukesh M, Harris E, Jena R, Evans P, Coles C. Relationship between irradiated breast volume and late normal tissue complications: a systematic review. Radiother Oncol 2012;104:1-10.

[11] Collette S, Collette L, Budiharto T, et al. Predictors of the risk of fibrosis at 10 years after breast conserving therapy for early breast cancer: a study based on the EORTC Trial 22881-10882 'boost versus no boost'. Eur J Cancer 2008;44: 2587-99.

[12] Offersen BV, Overgaard M, Kroman N, Overgaard J. Accelerated partial breast irradiation as part of breast conserving therapy of early breast carcinoma: a systematic review. Radiother Oncol 2009;90:1-13.

[13] Hurkmans CW, Dijckmans I, Reijnen M, van der Leer J, van Vliet-Vroegindeweij $C$, van der Sangen M. Adaptive radiation therapy for breast IMRTsimultaneously integrated boost: three-year clinical experience. Radiother Oncol 2012;103:183-7.

[14] Coles CE, Harris EJ, Donovan EM, et al. Evaluation of implanted gold seeds for breast radiotherapy planning and on treatment verification: a feasibility study on behalf of the IMPORT trialists. Radiother Oncol 2011;100:276-81.

[15] Al-Ghazal SK, Fallowfield L, Blamey RW. Does cosmetic outcome from treatment of primary breast cancer influence psychosocial morbidity? Eur J Surg Oncol 1999;25:571-3.

[16] Deasy JO, Bentzen SM, Jackson A, et al. Improving normal tissue complication probability models: the need to adopt a "data-pooling" culture. Int J Radiat Oncol Biol Phys 2010;76:S151-4.

[17] Barnett GC, Wilkinson JS, Moody AM, et al. Randomized controlled trial of forward-planned intensity modulated radiotherapy for early breast cancer: interim results at 2 years. Int J Radiat Oncol Biol Phys 2012;82:715-23. 
[18] Barnett GC, Wilkinson J, Moody AM, et al. A randomised controlled trial of forward-planned radiotherapy (IMRT) for early breast cancer: baseline characteristics and dosimetry results. Radiother Oncol 2009;92:34-41.

[19] Poortmans P, Bartelink H, Horiot JC, et al. The influence of the boost technique on local control in breast conserving treatment in the EORTC 'boost versus no boost' randomised trial. Radiother Oncol 2004;72:25-33.

[20] Deasy JO. Comments on the use of the Lyman-Kutcher-Burman model to describe tissue response to nonuniform irradiation. Int J Radiat Oncol Biol Phys 2000;47:1458-60.

[21] Gay HA, Niemierko A. A free program for calculating EUD-based NTCP and TCP in external beam radiotherapy. Phys Med 2007;23:115-25

[22] Jackson A, Ten Haken RK, Robertson JM, Kessler ML, Kutcher GJ, Lawrence TS Analysis of clinical complication data for radiation hepatitis using a parallel architecture model. Int J Radiat Oncol Biol Phys 1995;31:883-91.

[23] Roberts SA, Hendry JH. The delay before onset of accelerated tumour cell repopulation during radiotherapy: a direct maximum-likelihood analysis of a collection of worldwide tumour-control data. Radiother Oncol 1993;29:69-74.

[24] Yarnold J, Ashton A, Bliss J, et al. Fractionation sensitivity and dose response of late adverse effects in the breast after radiotherapy for early breast cancer: long-term results of a randomised trial. Radiother Oncol 2005;75:9-17.

[25] Yarnold J, Coles C. On behalf of the IMPORT LOW Trial Management Group. Intensity-modulated and partial organ radiotherapy. Randomised trial testing intensity-modulated and partial organ radiotherapy following breast conservative surgery for early breast cancer. Trial protocol, version 6; 2009, Institute of Cancer Research, Sutton, UK. p. 1-74. <http://clinicaltrials.gov/ct2/ show/NCT00814567>.

[26] Wolmark N, Curran W. On behalf of NSABP and RTOG of the American College of Radiology (ACR). NSABP Protocol B-39. RTOG Protocol 0413. A randomized phase III study of conventional whole breast irradiation versus partial breast irradiation for women with stage 0 , I, or II breast cancer. National surgical adjuvant breast and bowel project (NSABP). Trial protocol March 13, 2007. p. 1-132. <http://clinicaltrials.gov/ct2/show/NCT00103181>.

[27] van Luijk P, Delvigne TC, Schilstra C, Schippers JM. Estimation of parameters of dose-volume models and their confidence limits. Phys Med Biol 2003;48: 1863-84.

[28] Yorke ED. Modeling the effects of inhomogeneous dose distributions in normal tissues. Semin Radiat Oncol 2001;11:197-209.
[29] Myung J. Tutorial on maximum likelihood estimation. J Math Psychol 2003:47:90-100.

[30] Fehlauer F, Tribius S, Holler U, et al. Long-term radiation sequelae after breastconserving therapy in women with early-stage breast cancer: an observational study using the LENT-SOMA scoring system. Int J Radiat Oncol Biol Phys 2003:55:651-8

[31] Johansen J, Overgaard J, Rose C, et al. Cosmetic outcome and breast morbidity in breast-conserving treatment - results from the Danish DBCG-82TM national randomized trial in breast cancer. Acta Oncol 2002;41:369-80.

[32] Rancati T, Fiorino C, Gagliardi G, et al. Fitting late rectal bleeding data using different NTCP models: results from an Italian multi-centric study (AIROPROS0101). Radiother Oncol 2004;73:21-32.

[33] Mukesh MB, Barnett G, Cumming J, et al. Association of breast tumour bed seroma with post-operative complications and late normal tissue toxicity: results from the Cambridge Breast IMRT trial. Eur J Surg Oncol 2012;38: 918-24.

[34] Azria D, Gourgou S, Sozzi WJ, et al. Concomitant use of tamoxifen with radiotherapy enhances subcutaneous breast fibrosis in hypersensitive patients. Br J Cancer 2004:91:1251-60.

[35] Vrieling C, Collette L, Fourquet A, et al. The influence of patient, tumor and treatment factors on the cosmetic results after breast-conserving therapy in the EORTC 'boost vs. no boost' trial. EORTC Radiotherapy and Breast Cance Cooperative Groups. Radiother Oncol 2000;55:219-32.

[36] Immink JM, Putter H, Bartelink $H$, et al. Long-term cosmetic changes after breast-conserving treatment of patients with stage I-II breast cancer and included in the EORTC 'boost versus no boost' trial. Ann Oncol 2012;23: 2591-8.

[37] Bentzen SM, Overgaard M. Relationship between early and late normal-tissue injury after postmastectomy radiotherapy. Radiother Oncol 1991:20:159-65.

[38] Barnett GC, Wilkinson JS, Moody AM, et al. The Cambridge Breast Intensitymodulated Radiotherapy Trial: patient- and treatment-related factors that influence late toxicity. Clin Oncol (R Coll Radiol) 2011;23:662-73.

[39] Goldsmith C, Haviland J, Tsang Y, Sydenham M, Yarnold J. Large breast size as risk factor for late adverse effects of breast radiotherapy: is residual dose in homogeneity, despite 3D treatment planning and delivery, the main explanation? Radiother Oncol 2011;100:236-40. 